Research Article

\title{
Site Prediction Model for the over Rooftop Path in a Suburban Environment at Millimeter Wave
}

\author{
Young Keun Yoon (D), Kyung Won Kim, and Young Jun Chong
}

Electronics and Telecommunications Research Institute, Daejeon, Republic of Korea

Correspondence should be addressed to Young Keun Yoon; ykyoon@etri.re.kr

Received 27 July 2018; Revised 30 January 2019; Accepted 17 February 2019; Published 17 April 2019

Academic Editor: Rocco Guerriero

Copyright (c) 2019 Young Keun Yoon et al. This is an open access article distributed under the Creative Commons Attribution License, which permits unrestricted use, distribution, and reproduction in any medium, provided the original work is properly cited.

\begin{abstract}
This paper proposes the site-specific pathloss model for a small town in a suburban environment at millimeter wave. Also, the site general characteristics are provided in the entire measurement area of the small town. The proposed pathloss model is based on the moving measurement campaign according to the distance between a transmitter and a receiver in the candidate millimeter wave band or future five-generation service. In addition, the probability characteristics of pathloss such as the cumulative density function are shown to estimate the spreading characteristics of the wideband signal with a five-hundred-MHz bandwidth. Measurement was performed for the small town in a suburban covered with low-rise commercial restaurants or houses. Transmitting signals propagate through the over rooftop of low-rise houses of average ten meters height. Their multipath signals may be arrived at a receiving station located at the below roof of the house by reflection, diffraction, and scattered mechanism. A current propagation model for the over rooftop is based on generic or geometrical optic equations in a fully nonline-of-sight environment. But, non-line-of-sight cases in a realistic suburban environment include partly the open space between houses. Therefore, the definition of the propagation model of this sight-specific case is very important so clearly as to show the difference for the over rooftop according to the different displacements of houses in a non-line-of-sight environment. Finally, we suggest the improved propagation model of pathloss that can reflect various environments in a small town.
\end{abstract}

\section{Introduction}

Feasibility studies are currently being conducted on the millimeter frequency band over $20 \mathrm{GHz}$ [1-5]. On the other hand, the collected measurement and analysis data is still lacking. The millimeter wave (mm-Wave) spectrum provides greater bandwidth than $400 \mathrm{MHz}$ over commercial applications, but the propagation of $\mathrm{mm}$-Wave signals may be much less favorable. In high-density deployments, a wideband $\mathrm{mm}$-Wave signal can provide an alternative to cell segmentation by increasing the capacity of each hotspot cell. Due to the limited propagation range of $\mathrm{mm}$-Wave signals, future mobile applications will focus on hotspot cells in outdoor urban and suburban environments. The focus of this paper is on the urban low-rise (suburban) deployments with cell radiuses ranging from 63 to $514 \mathrm{~m}$. Measurement-based studies take into account actual Tx and Rx positions. Measurements were performed at regular Rx locations to collect statistics for various wireless parameters such as the pathloss factors and multipath components. You can use this information to understand the actual coverage of cells operating in the millimeter frequency band and perform system-level simulations based on the experience propagation model.

A recommendation in [6], which is based on the EUROCOST231 Walfisch-Ikegami model in [7], provides a shortdistance pathloss estimation method within $1 \mathrm{~km}$ and includes the site-general and site-specific pathloss model for the over rooftop propagation. On the site-general model, the applicable frequency range and environment is limited up to $66.5 \mathrm{GHz}$ in an urban environment. On the sitespecific model based on the geometrical optic (GO) mechanism, the applicable frequency range is limited up to $16 \mathrm{GHz}$ in an urban environment and up to $38 \mathrm{GHz}$ in a suburban area. However, the recommendation does not provide the propagation prediction model in the suburban area on the site-general characteristics and in the suburban area on 


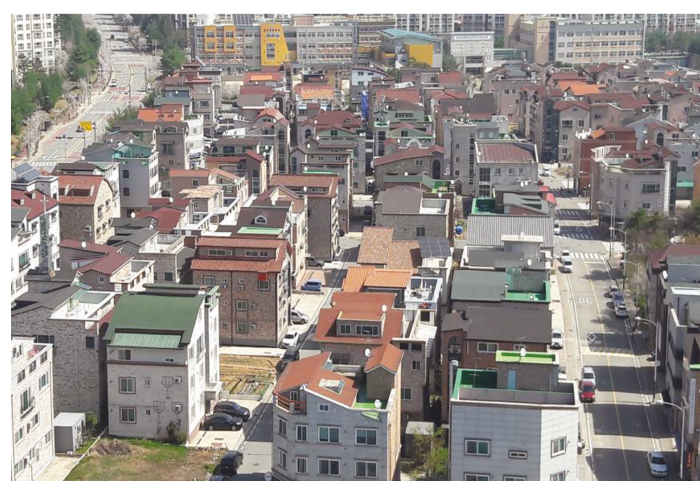

FIGURE 1: Small town in a suburban environment.

the site-specific characteristics including partly the open space between houses. Therefore, the estimation error of recommendation is too large to be useful for a realistic suburban environment including partly the open space between houses [8]. In [9], the pathloss prediction model for the over rooftop propagation in the suburban area at the microwave band is based on the propagation mechanism explained by using the GO and consists of the direct wave region, the reflected wave region, and the diffracted wave region. The boundary points of these regions can be determined uniquely from the antenna height and house environment, and all regions are continuously connected. Hence, the estimation of pathloss can be predicted from the short-distance line-of-sight region to the far-field non-line-of-sight region. Approaches to prediction of the over rooftop buildings apply to the residential of the city [10]. All buildings were assumed to be of the same height. The average received signals depend on transmitter antenna height and multigeometric diffraction statistics. In [11], when the receiver below a single building measures the signal power and the transmitter is much higher than the building roof but lower than the satellite, such as the unmanned aerial vehicle (UAV) communication link, the site-specific pathloss prediction applied a single knife-edge diffraction model based on the GO mechanism. Basic propagation elements such as reflection, diffraction, and scattering produce multipath propagation. In [12], multipath propagation effects predicted the wireless channel characteristics using the 2-ray or 3-ray simulation analysis based on the ray tracing mechanism in the time or frequency domain. In [13-15], various propagation models such as 2ray ground pathloss model, ITU-R street canyon model, and combined pathloss and shadowing model were used to evaluate the impact on system performance in mobile networks. In addition to this, the use of any propagation model has a significant impact on the performance of the wireless communication network [16]. In [17, 18], a measurementbased analysis of $38 \mathrm{GHz} \mathrm{mm}$-Wave propagation in urban areas has been conducted with narrow beamwidth antennas and focused on the characterization of various basic factors, which are required in the propagation model, such as the line of sight, reflection, diffraction, and transmission. Based on ray tracing using a simplified $3 \mathrm{D}$ geometric description of the propagation environment, the map-based channel model

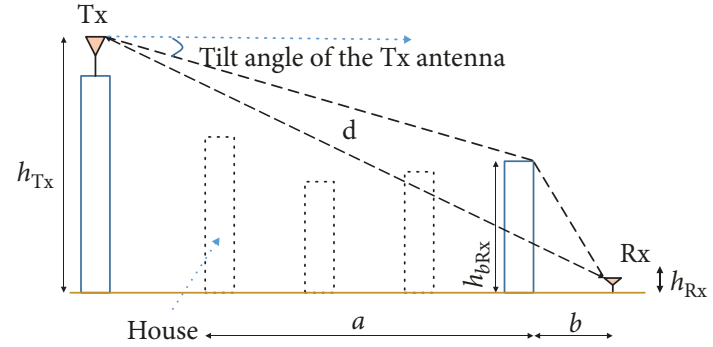

Figure 2: Side view of the house geometry and parameters.

was presented at six different frequencies between 2 and $60 \mathrm{GHz}[19]$.

However, existing data and forecasting methods do not reflect the realistic environments with partially open spaces between houses in a suburban small town. To provide a realistic assessment of $\mathrm{mm}$-Wave propagation in a suburban area, we estimated using site-specific modeling and conducted measurements on the $32.4 \mathrm{GHz}$ channel. The pathloss model for the over rooftop propagation in the $\mathrm{mm}$-Wave band considering the above concerns is proposed for the $5 \mathrm{G}$ generation service. The proposed pathloss model consists of three parts: the direct wave dominant region, the reflected dominant region, and the diffracted dominant region.

\section{Measurement Campaign}

2.1. Measurement Environment and Scenario. In high-density deployments, the wideband of the $\mathrm{mm}$-Wave signal can provide an alternative to the cell plan by greatly increasing the capacity of individual small cells. Because the mm-Wave system consists of small cells, the relative pathloss and the cell coupling change quickly. From a system point of view, this means that connectivity is very intermittent and communications must be able to adapt quickly. Due to the limited range of the mm-Wave signals, most cellular applications in the mm-Wave system focus on small cells with the cell radius of $100 \mathrm{~m}$ or $500 \mathrm{~m}$ in the outdoor urban environment. The channel measurement sites based on the urban low-rise (suburban) environment are carefully selected. During measurement, the location of the transmitting antenna is fixed on the roof of a tall building, so you should be able to see over the roof of every house in the whole measuring area. Measurement environments include low-rise houses, grid roads, multiple vehicles, and auxiliary facilities. There are two types of wireless links when working locally in a small town environment with regular grid roads except for some road blocks. One is a line-of-sight (LoS) link, and the other is a non-line-of- sight (NLoS) link. When Rx is covered by houses or obstacles and the transmit antenna of Tx is not visible at the $\mathrm{Rx}$ location, the NLoS link occurs. Measurement was carried out in a small town of the city.

The characteristics of a small town are shown in Figure 1. A small town with an urban low-rise (suburban) environment shows the layout of regular houses with three or four floors. And the roof of the house is sloping or flat-type.

As shown in Figure 2, a transmitter (Tx), mounted on a $2 \mathrm{~m}$ high tripod, is installed on a roof with the height $\left(h_{\mathrm{Tx}}\right)$ 


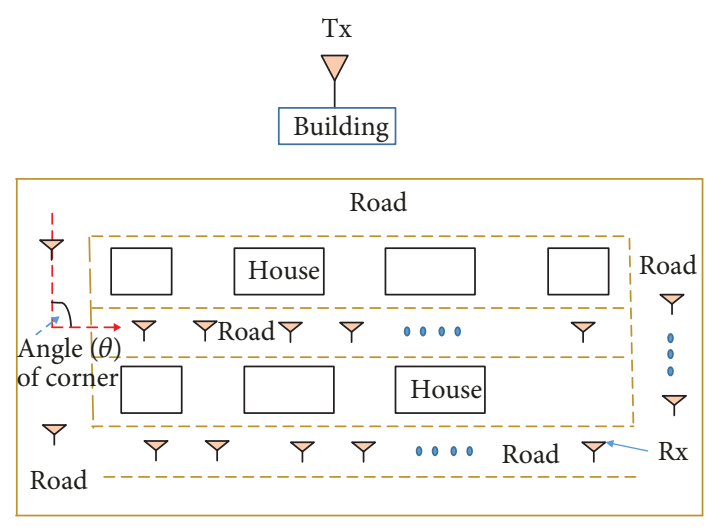

FIgURE 3: Top view of the house and road geometry.

of $42 \mathrm{~m}$ above the ground level. A receiver ( $\mathrm{Rx})$ is at a height $\left(h_{R \mathrm{x}}\right)$ of $1.5 \mathrm{~m}$ above the ground level. Tx and $\mathrm{Rx}$ are the single-frequency modes with an occupied bandwidth (BW) of $500 \mathrm{MHz}$. The center of operating frequency is $32.4 \mathrm{GHz}$. The maximum Tx output power $(P \mathrm{Tx})$ is $27 \mathrm{dBm} / \mathrm{BW}$. The antennas of Tx and Rx are a type of aperture horn with a directional gain.

In Figure 2, the horn antenna direction of $\mathrm{Tx}$ is tilted at an angle range of -4 to -37 degrees from Tx to the roof of the house so that the Tx beam covers the measurement area where the receiving sensitivity of the signal-to-noise ratio $(\mathrm{S} / \mathrm{N})$ to noise floor is more than $10 \mathrm{~dB}$. The closer the $\mathrm{Tx}$ is to the house, the greater the tilt angle of the $\mathrm{Tx}$ antenna. The Tx horn antenna has a 15-degree beamwidth and a $21 \mathrm{dBi}$ directional gain. The horn antenna of $\mathrm{Rx}$ is the same as the horn antenna of Tx. This is the reason that the pathloss of the mm-Wave band is large. To estimate a large pathloss, the path gain was obtained using a horn antenna with a large directional gain. With respect to antenna characteristics, the far-field antenna pattern and gain characteristics in the mm-Wave band were measured in the anechoic chamber.

In Figure 3, in the path of the over rooftop environment, Rx moved around the house or along the road in the whole measurement area and measured the channel impulse response at the location of the Rx measurement point. The over rooftop path environment refers to an outdoor environment where the transmitted signal passes above the roof of the house and is received at the Rx measurement point. However, due to the large entry loss of the house with a reinforced concrete structure in mm-Wave, this paper did not consider the penetration loss of house [20-22]. When looking at Tx at the $\mathrm{Rx}$ position, the Tx site is visible or invisible. In this case, it means LoS or NLoS, respectively.

2.2. Hardware Channel Sounder for Measurement. We built a wideband measurement sounder that operates at the center frequency of $32.4 \mathrm{GHz}$ and $500 \mathrm{MHz}$ BW, capable of detecting multipath signals around an urban low-rise (small town in suburban) outdoor environment. Measured multipath signals have temporal or spatial variation characteristics.

For the performance of the measurement sounder, the measurement time resolution is $2 \mathrm{~ns}$, depending on the occupied bandwidth of the system. The spatial dynamic range from which the received signal can be measured and recovered is approximately $150 \mathrm{~dB}$ to provide a sufficient margin for a noise floor of approximately $-78 \mathrm{dBm} / \mathrm{BW}$ in the analysis.

The measurement sounder transmits the conducted power of $27 \mathrm{dBm} / \mathrm{BW}$. It can be amplified by the directional antenna. The best antenna pair of Tx and Rx is selected by referring to the characteristics of the multipath signals around the small town.

In $\mathrm{mm}$-Wave bands from 20 to $40 \mathrm{GHz}$, the gain of a commercial directional antenna is less than about $25 \mathrm{dBi}$.

As shown in Figure 4, the measurement sounder includes the following modules. Rx has the same structure except for the radio frequency $(\mathrm{RF})$ module of $\mathrm{Tx}$.

(i) Antenna and radio frequency (antenna/RF) module, which up-converts the intermediate frequency (IF) signal to an RF signal of $32.4 \mathrm{GHz}$. The output port connects a directional antenna. The IF signal is received through the input port. The antenna and $\mathrm{RF}$ module are interconnected and can be separated

(ii) Antenna/RF rotation module, which measures the multipath signals received from various angles by rotating the antenna/RF module

(iii) A timing synchronization and signal processing module, which synchronizes the start time between $\mathrm{Tx}$ and $\mathrm{Rx}$ in units of timing, including baseband or IF signal processing capabilities

(iv) Monitoring module, which provides input parameters for overall control and monitors the output signal and received signal characteristics of each module

\section{Measurement and Results}

3.1. Channel Impulse Response. Multipaths that result in intersymbol interference cause time dispersion as described in the channel impulse response (CIR). When the channel characteristics change over time, a time $t$ is required, and $\tau$ is the multipath delay. Let equivalent complex-envelop CIR in a time variant at $\mathrm{RX}$ be

$$
h(t, \tau)=\sum_{i=0}^{I-1} p_{i}(t, \tau) \exp \left(-j \varphi_{i}(t, \tau)\right) \delta\left(\tau-\tau_{i}(t)\right),
$$

where $p_{i}, \varphi_{i}, \tau_{i}$ are the amplitude, phase shift, and excess delay of the $i$-th propagation path, respectively. I means total multipath components of the transmitted signal arriving at $\mathrm{Rx}$. Useful to discrete $\tau$ into delay profiles, each profile represents a multipath component. At time $t$, each profile arrives at $\mathrm{Rx}$ with a different amplitude, goes through a different phase shift, and has a different excess delay. To derive the received signal strength intensity (RSSI) at $\mathrm{Rx}$, it is assumed that the channel characteristic does not change with time. This means that multipath components of transmitted signals arriving at the same $\mathrm{Rx}$ location have the same time 


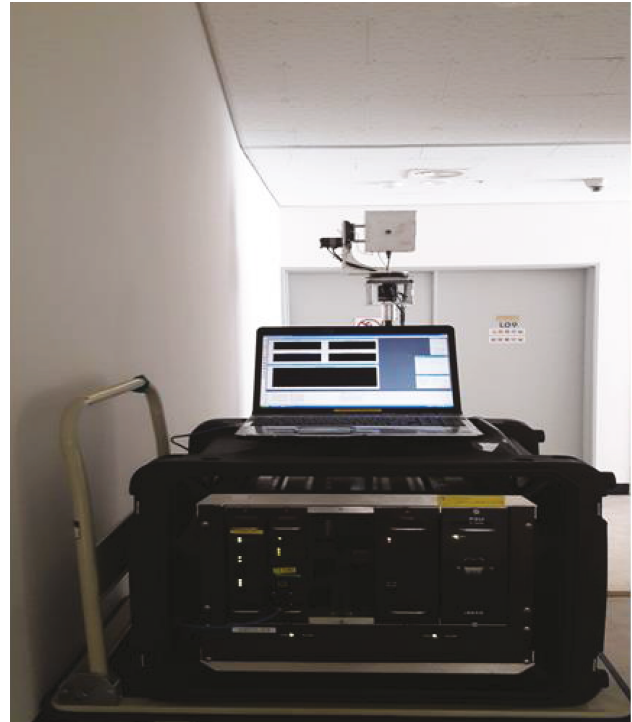

(a)

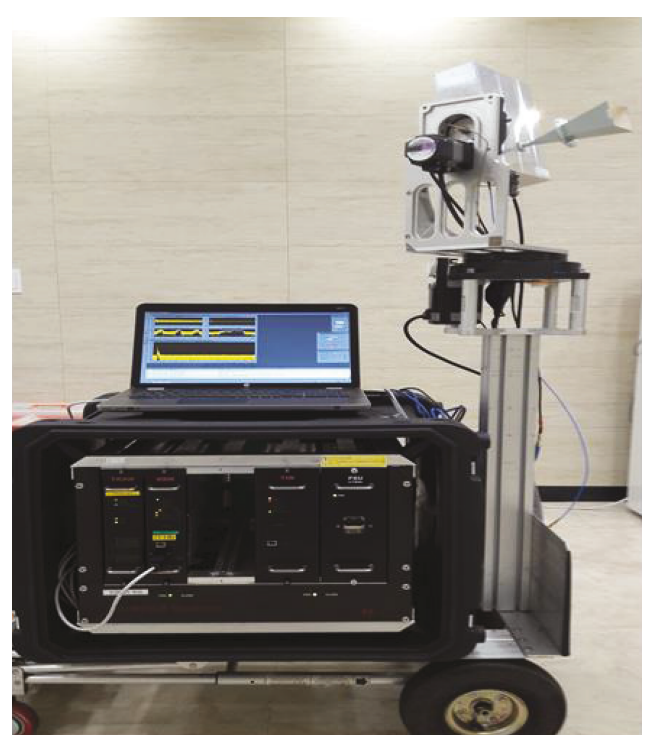

(b)

FIGURE 4: Propagation channeling sounder (transmitter (a) and receiver (b)).

excess delay. Let equivalent complex-envelop CIR in the time invariant at $\mathrm{RX}$ be

$$
h(\tau)=\sum_{i=0}^{I-1} p_{i} \exp \left(-j \varphi_{i}\right) \delta\left(\tau-\tau_{i}\right)
$$

3.2. Received Signal Strength Intensity. At any frequency band and $\mathrm{Rx}$ position, the RSSI of the transmission and reception distance is the received $\mathrm{Rx}$ power derived using the channel response characteristic of Eq. (2). The RSSI depends on the separated distance ( $\mathrm{Tx}-\mathrm{Rx}$ distance) between $\mathrm{Tx}$ and $\mathrm{Rx}$. Generally, as the distance increases, the received power decreases. In particular, the higher the frequency is, the greater the path attenuation characteristics are. The link distance from $\mathrm{Tx}$ to $\mathrm{Rx}$ is one of the major problems facing the mm-Wave system. In this paper, we considered an important factor in evaluating the coverage performance of the $\mathrm{mm}$-Wave system. The application range of the mm-Wave system is to determine how the magnitude of the received $\mathrm{Rx}$ power varies with the transmission and reception distances.

$$
\mathrm{RSSI}=P \mathrm{Tx}+G(\mathrm{Tx}, \mathrm{Rx})-\mathrm{PL} .
$$

Here, RSSI, $P$ Tx, $G(\mathrm{Tx}, \mathrm{Rx})$, and PL are the received signal strength intensity, the Tx output power, the Tx and Rx antenna gain, and pathloss, respectively.

3.3. General Approach on Pathloss Prediction. In Eq. (3), the pathloss in [6] can be calculated theoretically by knowing the Tx output power, the Tx and Rx antenna gains, and the $\mathrm{Rx}$ receive power. However, the pathloss characteristics can be derived by Eq. (4) according to frequency, the separated distance between $\mathrm{Tx}$ and $\mathrm{Rx}$, and the long-term fading (shadow fading) characteristics. In Eq. (4), the exponents of the coefficient according to each parameter should be able to be derived from the actual environmental measurement.

$$
\operatorname{PL}(d, f)=10 \alpha \log _{10}(d)+\beta+10 \gamma \log _{10}(f)+N(0, \sigma),
$$

where

$d: 3 \mathrm{D}$ distance between $\mathrm{Tx}$ and $\mathrm{Rx}(\mathrm{m})$

$f$ : operating frequency $(\mathrm{GHz})$

$\alpha$ : coefficient associated with the increase in pathloss with the distance

$\beta$ : coefficient associated with the offset value of pathloss with multiple frequencies

$\gamma$ : coefficient associated with the increase in pathloss with multiple frequencies

$N(0, \sigma)$ : zero mean Gaussian random variable with a standard deviation $\sigma(\mathrm{dB})$

The international recommendation for pathloss estimates for short distances of up to $1 \mathrm{~km}$ is the standardization of ITU-R (ITU radio sector) P.1411 [6]. This prediction model of pathloss is based on the measured data, and it is possible to predict pathloss considering both LoS and NLoS in the rooftop environment.

To determine the probability distribution characteristics of the entire measurement area for pathloss, we derived the cumulative distribution function (CDF) to determine the relative differences in the path characteristics of LoS or NLoS or opened area. The CDF of a random variable represents the distribution of a random variable. The advantage of CDF is that it can be defined for all kinds of random variables (discrete, continuous, and mixed). The $\mathrm{CDF}$ of random variable $X$ is defined as

$$
F_{X}(x)=P(X \leq x) \text { for all } x \in \mathbb{R} \text {. }
$$

Let $X$ be the number of observed minimum pathloss values. 


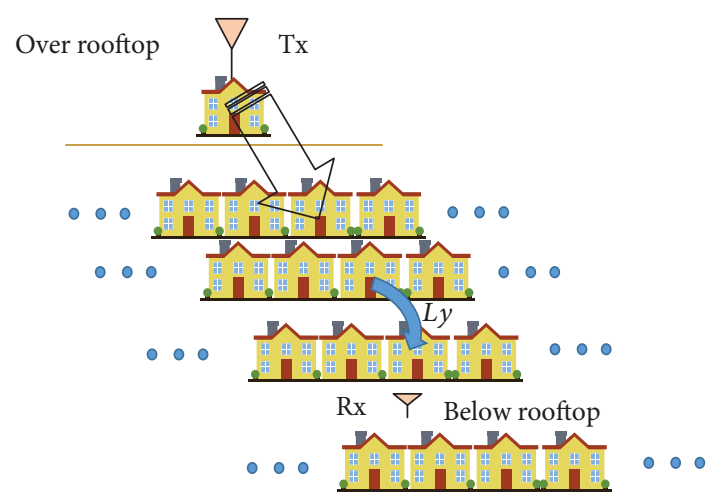

Figure 5: A case that houses are stuck together in the over rooftop environment.

In general, let $X$ be a discrete random variable with the range $R_{X}=\left\{x_{1}, x_{2}, x_{3}, \cdots\right\}$, such that $x_{1}<x_{2}<x_{3}, \cdots$. Here, we assume that the range $R_{X}$ is bound from below; i.e., $x_{1}$ is the smallest value in $R_{X}$. If this is not the case, then $F_{X}(x)$ approaches zero as $x \rightarrow-\infty$ rather than hitting zero. We see that the CDF is in the form of a staircase. In particular, note that the CDF starts at zero. Then, it jumps at each point in the range. The CDF is always a nondecreasing function; i.e., if $y \geq x$, then $F_{X}(y) \geq F_{X}(x)$. Finally, the $\mathrm{CDF}$ approaches 1 as $x$ becomes large. Note that the CDF completely describes the distribution of a discrete random variable.

3.4. Site-Specific Environments. As shown in Figures 5 and 6, we considered the different site-specific characteristics for a small town to evaluate and analyze pathloss with the distance in the over rooftop environment.

The figures show that the radio waves propagate through the path between Tx on the top floor of the house and $\mathrm{Rx}$ below the roof. Figure 5 shows the case where the houses are stuck together, and Figure 6 shows the small town environment where the houses are separated from each other as shown in Figure 1. As a numerical model for the over rooftop environment, the international recommendation ITU-R P.1411 related to loss estimation with regard to short distance is given on the basis of the GO mechanism and related in the Figure 5 case. However, there is no specific model for the over rooftop environments considering spaces and along roads between houses. If the house is far away, the arrival path of the radio should take into account the signal coming from the road at the Rx point, the signal coming from the interhouse space, and the signal coming from above the house as shown in Figure 6. However, existing models that take into account the environment in Figure 5 only consider signals passing over the roof of the house. This is because it assumes an infinite array of houses.

\subsection{Measurement Results Based on a Site-Specific} Environment. The site-specific environments in a small town are subdivided into both free space and three cases in a LoS or NLoS environment.

The routes of the signals passing above houses are divided into three paths when the adjacent houses are slightly apart as shown in Figure 6. The first is a route that travels around the corner along the road (Path $\left.L_{r}\right)$. The second is a path coming from inter-house space (Path $L_{b}$ ). The third is a route that passes over the house roof (Path $L_{v}$ ).

We conducted the measurement for the propagation of the over rooftop in the mm-Wave bands in an urban lowrise (suburban) environment. We found that the measurement results for the site-specific environment in Figure 6 were different with the existing prediction result of the GO basis for the site-specific environment in Figure 5. This reason is a difference in the arrangement and the length of houses. In Figure 5, the length of the house is finite, but the arrangement between houses is stuck together. On the other hand, the considered environment in Figure 6 includes an open-space area between houses and neighboring houses. As a result, the existing GO basis prediction results do not fully reflect the characteristics of various small town environments. Therefore, this paper proposes the novel prediction model that can improve the existing prediction models of pathloss. The following is about the measurement method.

In order to obtain the propagation characteristics according to the distance through measurement, the Rx measurement points were moved at multiple positions within the entire measurement area as shown in Figure 3. The measurement points are located along the road and are at the front, back, and side of the house. A high gain-received horn antenna was used for measurement. At each Rx measurement point, the $\mathrm{Rx}$ antenna module was rotated horizontally and vertically to measure multipath signals coming from various directions, such as reflection and diffraction around $\mathrm{Rx}$. And the measured CIR data is collected at each Rx measurement position. Both the Tx and Rx horn antennas have the same vertical polarization. From the measured data, pathloss according to each distance to the measurement point from Tx was derived by Eq. (2) to Eq. (4). In order to derive a predictive model for three paths in the site-specific environment as shown in Figure 6, the measured data was compared to the opened area characteristic [23].

On measurement, $\mathrm{Tx}$ is installed on the roof at the height of $44 \mathrm{~m}$ and $\mathrm{Rx}$ is located on the height of $1.5 \mathrm{~m}$ above the ground level. Tx and Rx have a single-frequency mode with the same frequency of $32.4 \mathrm{GHz}$. The occupied bandwidth is $500 \mathrm{MHz}$. The maximum Tx output power is $27 \mathrm{dBm} / \mathrm{BW}$. $\mathrm{Tx}$ and $\mathrm{Rx}$ antennas are of the same type of aperture horn with a directional gain of $21 \mathrm{dBi}$. The beamwidth of the horn antenna is 15 degrees. The small town includes a LoS or NLoS environment. The maximum 3D distance from the Tx site in the entire measurement area is less than $500 \mathrm{~m}$. The minimum distance is less than $98 \mathrm{~m}$.

The following shows the distribution of the measured data according to the distance for the three paths in the over rooftop of the house. The regression lines were drawn based on the measured data. Pathloss when the frequency is a single frequency can be expressed as Eq. (6) from Eq. (4). From the regression line, the coefficients $(\alpha, \delta)$ of Eq. (6) can be derived.

$$
\operatorname{PL}(d)=10 \alpha \log _{10}(d)+\delta
$$

where 


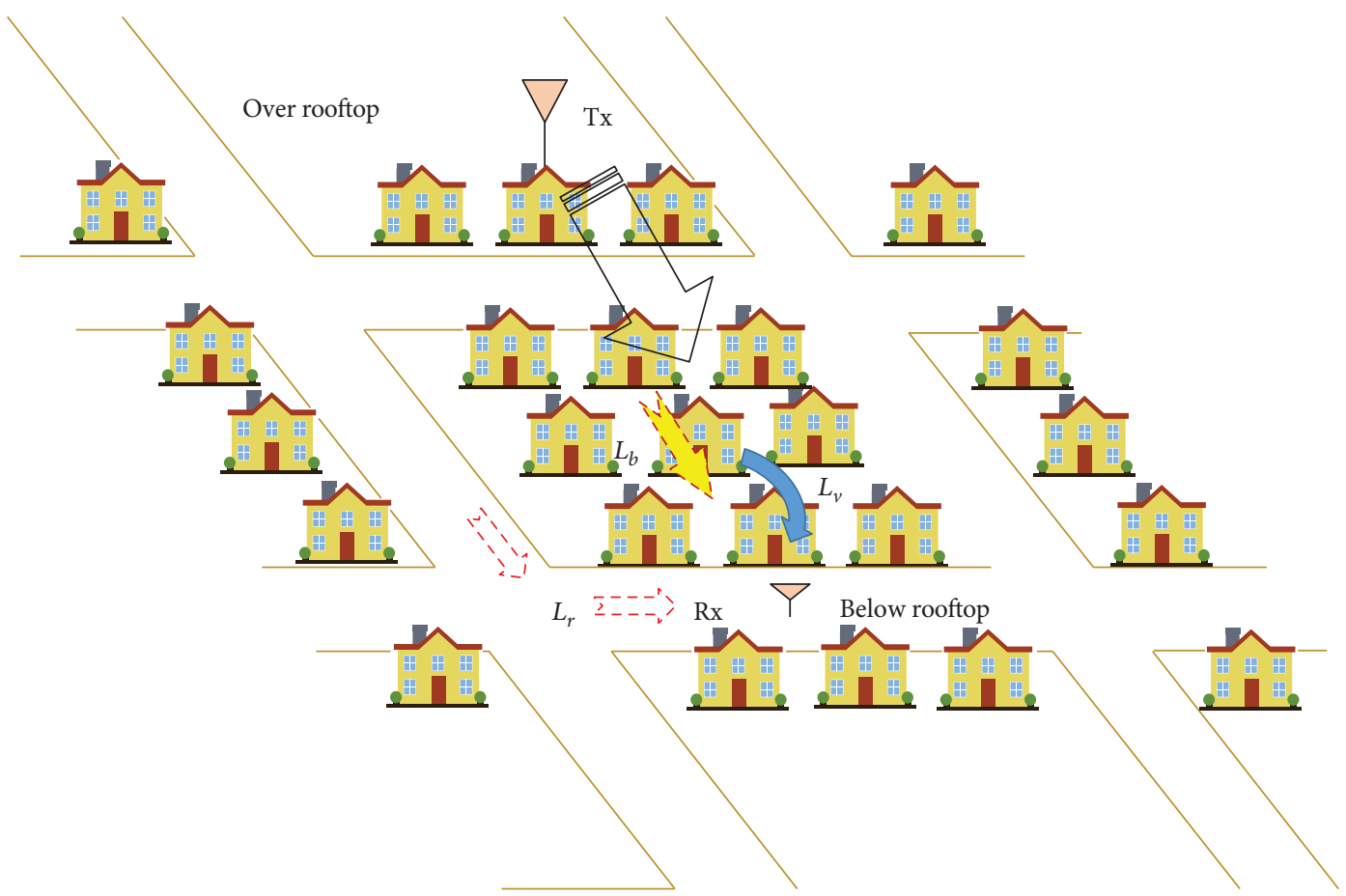

Figure 6: A case that houses are separated together in the over rooftop environment.

$d$ : 3D distance between $\mathrm{Tx}$ and $\mathrm{Rx}(\mathrm{m})$

$\alpha$ : coefficient associated with the increase in pathloss with the distance

$\delta$ : coefficient associated with the offset value of pathloss in single frequency

The first of three paths is a route that travels around the corner along the road (Path $L_{r}$ ). This path is divided into the path (the path before corner) from the Tx site to the corner and the path (the path after corner) from the corner to the Rx location. On the path before the corner, LoS and NLoS environments coexist in the path until the corner. Figure 7 shows the measured data and path characteristics according to the distance from the Tx site to the corner (the path before corner). The measured data was compared to the opened area characteristic. We show pathloss according to the distance by separating measurement points by LoS (Meas. LOS-Lrbc-road) or NLoS (Meas.NLOS-Lrbc-road) environment on the path. The regression lines in a LoS or NLoS environment were drawn based on the measured data. Table 1 shows the coefficients derived from the regression lines in Figure 7. The coefficients associated with pathloss are different depending on the LoS or NLoS environment. That is, in the case of the NLoS environment, the relative pathloss is greater than LoS, and the slope with respect to pathloss is also increased. The pathloss factor according to the distance seems larger than the opened area loss in case of LoS, and the relative pathloss in NLoS is about $27 \mathrm{~dB}$ larger than LoS.

Typical pathloss parameters estimated from all power profiles for an outdoor environment are given in Table 1. The probability values of $50 \% \mathrm{CDF}$ for pathlosses at $32.4 \mathrm{GHz}$ for the before-corner path in a small town are shown in Figure 8. For the outdoor environment, pathlosses are dependent on the opened area or LoS or NLoS

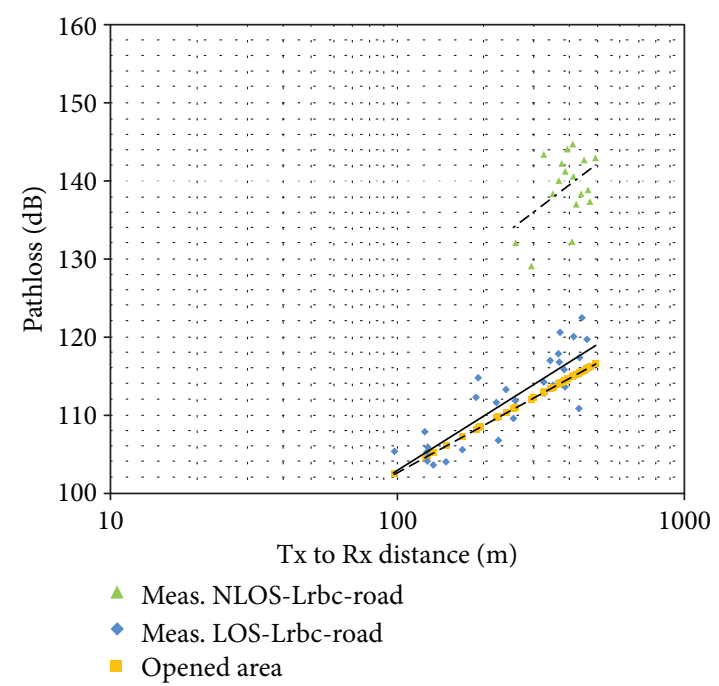

Figure 7: Pathloss characteristics according to the distance on the route that travels before the corner along the road (at site-specific).

environment as shown in results in Figure 8. On the route that travels before the corner along the road from the Tx site, median pathloss values of the opened area and LoS and NLoS environments are $110 \mathrm{~dB}, 113 \mathrm{~dB}$, and $139 \mathrm{~dB}$, respectively.

The second is a path coming from the inter-house space (Path $L_{b}$ ). On the inter-house space path, LoS and NLoS environments coexist on the path until Rx. Figure 9 shows the measured data and the path characteristics according to the distance when the transmitting signals pass through the inter-house space on the received path. The measured data was compared with the opened area characteristic. We show pathloss according to the distance by separating 
TABLE 1: Pathloss coefficient on the route that travels before the corner along the road (at site-specific).

\begin{tabular}{lccrrr}
\hline Frequency $(\mathrm{GHz})$ & Distance range $(\mathrm{m})$ & Type of environment & LoS/NLoS & $\alpha_{1}$ & $\delta_{1}$ \\
\hline \multirow{3}{*}{32.4} & $98-492$ & Opened area & LoS & 2.0 & 62.7 \\
& & Urban low-rise (Meas.LOS-Lrbc-road) & 57.0 \\
& $300-492$ & Urban low-rise (Meas.NLOS-Lrbc-road) & NLoS & 2.8 & 66.8 \\
\hline
\end{tabular}

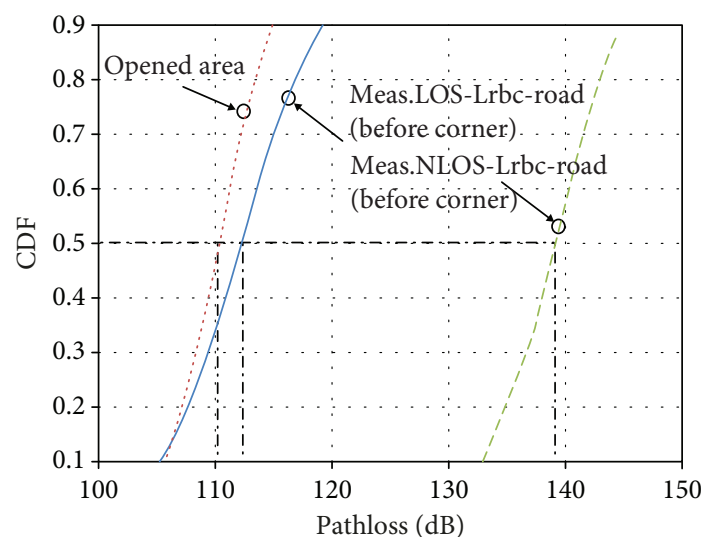

FIGURE 8: CDF on the route that travels before the corner along the road (at site-specific).

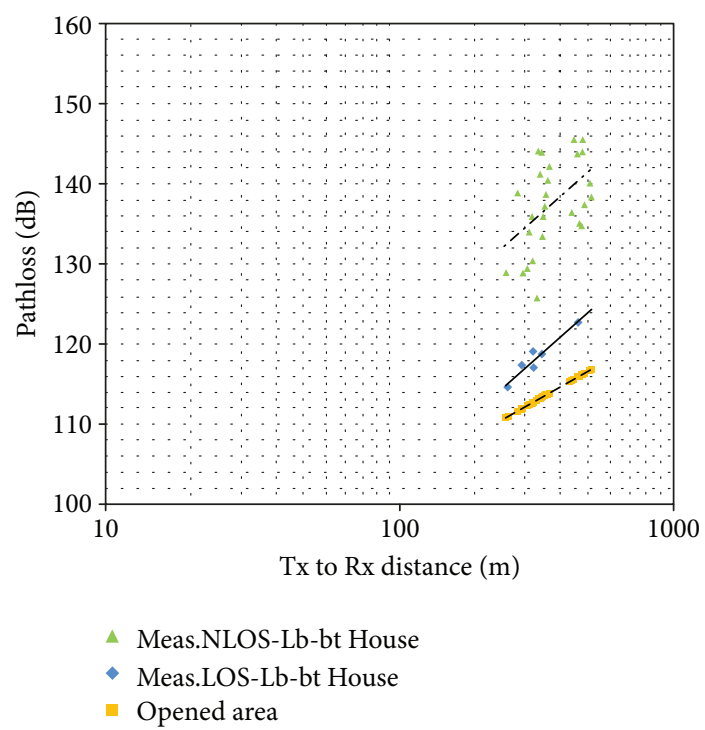

FIgURe 9: Pathloss characteristics according to the distance on a path coming from inter-house space (at site-specific).

measurement points by a LoS (Meas.LOS-Lb-btHouse) or NLoS (Meas.NLOS-Lb-btHouse) environment on the path. The regression lines in LoS and NLoS environments were drawn based on the measured data, too.

Table 2 shows the coefficient of parameters derived from the regression lines in Figure 9. The coefficients associated with pathloss are different depending on the LoS or NLoS environment. That is, in the case of NLoS, the relative pathloss is greater than LoS, and the slope with respect to pathloss also increases similarly. The relative pathloss seems larger at $6 \mathrm{~dB}$ than the opened area loss in spite of the LoS environment, and the relative pathloss in NLoS is about $19 \mathrm{~dB}$ larger than that in LoS. Typical pathloss parameters estimated from all power profiles for an outdoor inter-house space environment are given in Table 2 .

The probability values of $50 \%$ CDF for pathlosses for the between-house path are shown in Figure 10. Pathlosses are dependent on the opened area and LoS and NLoS environments as shown in results in Figure 9. On the path coming from the inter-house space, median pathloss values of the opened area and LoS and NLoS environments are $113 \mathrm{~dB}$, $119 \mathrm{~dB}$, and $138 \mathrm{~dB}$, respectively.

The third is a route that passes over the house roof (Path $L_{v}$ ). On the path over the house roof, the path until $\mathrm{Rx}$ is the NLoS environment.

Figure 11 shows the measured data and path characteristics depending on the distance when transmitting signals pass over the house roof on the received path. The measured data was compared with the opened area and the inter-house space path characteristics. We show pathloss according to the distance by separating measurement points by the path of inter-house space (Meas.NLOS-Lb-btHouse) or the path over the house roof (Meas.NLOS-Lv-over House Roof) environment. The regression lines in two environments were also drawn based on the measured data.

Table 3 shows the coefficient of parameters derived from the regression lines in Figure 11. The coefficients associated with pathloss are different depending on the opened area or NLoS environment. That is, in the case of NLoS, the relative pathloss is greater than the opened area, and the slope with respect to pathloss also increases sharply. The relative pathloss seems larger $3 \mathrm{~dB}$ than the inter-house space loss, and the relative pathloss in NLoS is about $26 \mathrm{~dB}$ larger than the opened area.

Typical pathloss parameters estimated from all power profiles of the path over the house roof are given in Table 3. The probability values of $50 \%$ CDF for pathlosses for the over house roof path are shown in Figure 12. Pathlosses are dependent on the NLoS environment as shown in results in Figure 12. On the route that passes over the house roof, median pathloss values of NLoS environments are $139 \mathrm{~dB}$.

\section{Modeling Based on Measurement}

4.1. Proposal of a Site-Specific Prediction Model. For the environment in Figure 5, the existing pathloss prediction model based on the GO is divided into three parts: the direct wave dominant region, the reflected wave dominant region, and the diffracted wave dominant region. This model can predict the loss including LoS and NLoS. However, due to the diversity of the site environment, it can be seen that the existing model has limitations that can be applied as predictive model in outdoor environments on the over rooftop paths related to 
the arrangement of infinite houses. Therefore, in this paper, we propose the novel pathloss model that can be analyzed and predicted according to the arrangement of houses. This proposed model is based on the measured data. Due to the variety of measurement environments, predictable and environmentally relevant parameters can be applied.

Figure 6 describes a propagation characteristic that predicts whole pathloss between $\mathrm{Tx}$ and $\mathrm{Rx}$ of the low house height. The proposed pathloss model based on measurement is divided into three parts for outdoor environment: pathloss along a road, pathloss between houses, and pathloss above the house roof. Applicable areas are both LoS and NLoS regions that include areas having two or more corners. The pathloss along a road $\left(L_{r}\right)$ is dominant at a relatively nearby Tx where there are only a few corners and the pathloss between houses $\left(L_{b}\right)$ becomes dominant as a distance between terminals increases. The above roof propagation pathloss $\left(L_{v}\right)$ becomes dominant relatively far from Tx where $L_{b}$ increases by multiple shielding of the houses. This model is recommended for frequencies in the millimeter range around $30 \mathrm{GHz}$. The maximum distance between terminals $d$ is up to $500 \mathrm{~m}$. The applicable road angle range is below 90 degrees.

The following Eq. (7) to Eq. (16) are the site-specific pathloss models based on measurement and proposed in the over rooftop in an urban low-rise (suburban) environment.

In a direct wave dominant region,

$$
L=20 \log _{10}\left(\frac{4 \pi d}{\lambda}\right) \text { in opened area. }
$$

In a reflected wave dominant region,

$$
\begin{aligned}
& L=10 \log _{10}\left(\frac{1}{10^{\left(L_{\mathrm{r}} / 10\right)}}+\frac{1}{10^{\left(L_{\mathrm{b}} / 10\right)}}+\frac{1}{10^{\left(L_{v} / 10\right)}}\right), \\
& L_{r}= \begin{cases}L_{r \mathrm{bc}} & \text { (before corner) } \\
L_{r \mathrm{ac}} & (\text { after corner }),\end{cases} \\
& L_{r \mathrm{bc}}=10 \alpha_{1} \log _{10}(d)+\delta_{1} \text { inLos } / \text { NLoS } \\
& L_{\mathrm{rac}}=L_{\mathrm{rbc}}+\left(7.6 \log _{10}(\theta)+7.56\right) \\
& \cdot\left\{1-\exp \left(-3.72 \cdot 10^{-5} \theta x_{1} x_{2}\right)\right\} \text {, } \\
& L_{b}=20 \log _{10}\left(\frac{4 \pi d}{\lambda}\right)+10 \alpha_{2} \log _{10}(d) \\
& +\delta_{2} \text { in LoS/NLoS, } \\
& L_{v}=20 \log _{10}\left(\frac{4 \pi d}{\lambda}\right)+10 \alpha_{3} \log _{10}(d)+\delta_{3} \text { in NLoS. }
\end{aligned}
$$

In a diffracted wave dominant region,

$$
L=20 \log _{10}\left(\frac{4 \pi d}{\lambda}\right)+L_{1}
$$

$$
\begin{aligned}
& L_{1}=6.9+20 \log _{10}\left(\sqrt{\left(v_{1}-0.1\right)^{2}+1}+v_{1}-0.1\right), \\
& v_{1}=\left(h_{b \mathrm{Rx}}-h_{\mathrm{Rx}}\right) \sqrt{\frac{2}{\lambda}\left(\frac{1}{a}+\frac{1}{b}\right)} .
\end{aligned}
$$

The relevant parameters for this model are

$\lambda$ : wavelength $(\mathrm{m})$

$d$ : 3D distance between $\mathrm{Tx}$ and $\mathrm{Rx}(\mathrm{m})$

$\alpha_{1}$ : coefficient associated with the increase in pathloss with the distance along a corner

$\delta_{1}$ : coefficient associated with the offset value of pathloss along a corner in single frequency

$\alpha_{2}$ : coefficient associated with the increase in pathloss with the distance between houses

$\delta_{2}$ : coefficient associated with the offset value of pathloss between houses in single frequency

$\alpha_{3}$ : coefficient associated with the increase in pathloss with the distance above the roof of a house

$\delta_{3}$ : coefficient associated with the offset value of pathloss above the roof of a house in single frequency

$\theta$ : road angle of the corner (degree)

$f$ : operating frequency $(\mathrm{GHz})$

$x_{1}$ : distance from $\mathrm{Tx}$ to a corner $(\mathrm{m})$

$x_{2}$ : distance from a corner to $\mathrm{Rx}(\mathrm{m})$

$R$ : visible $3 \mathrm{D}$ distance from $\mathrm{Tx}$ to a house $(\mathrm{m})$

$h_{b \mathrm{Rx}}$ : height of the nearest house from $\mathrm{Rx}$ in Tx direction $(\mathrm{m})$

$h_{\mathrm{Rx}}: \mathrm{Rx}$ antenna height $(\mathrm{m})$

$a$ : distance between the nearest house from $\mathrm{Tx}$ and $\mathrm{Rx}(\mathrm{m})$

$b$ : distance between $\mathrm{Rx}$ and the nearest house from $\mathrm{Rx}(\mathrm{m})$

The prediction error of the proposed model can be deduced from the difference between the predicted and measurement values of each measurement position in an actual measurement environment. The root mean square error (RMSE) value in a real suburban environment is presented as the estimation error of the proposed model. The estimation error of the proposed model depends on the pathloss along a road $\left(L_{r}\right)$, the pathloss between houses $\left(L_{b}\right)$, and the above roof of propagation pathloss $\left(L_{v}\right)$. As results shown in Table 4, the estimation error of the proposed model is less than $5 \mathrm{~dB}$ in the millimeter wave band.

The proposed site-specific predication model based on real environment measurement can be usefully applied in some of the open space between houses, which cannot be analyzed in the existing P.1411 prediction model. As an environment basis prediction model, it is possible to derive sitespecific predication propagation characteristics according to the real environment considering building height, building width, building surface roughness, and different building materials. Also, in the case of the site-specific predication model using the GO basis simulation technique like the existing P.1411 model, there is a limitation in the portion of simulating the building characteristics for the real environment. The proposed model is able to overcome the limit of the existing simulation technique because it can predict the 
TABLe 2: Pathloss coefficient on a path coming from inter-house space (at site-specific).

\begin{tabular}{|c|c|c|c|c|c|}
\hline Frequency $(\mathrm{GHz})$ & Distance range $(\mathrm{m})$ & Type of environment & LoS/NLoS & $\alpha_{2}$ & $\delta_{2}$ \\
\hline \multirow{3}{*}{32.4} & \multirow{2}{*}{$260-460$} & Opened area & \multirow{2}{*}{ LoS } & 2.0 & 62.7 \\
\hline & & Urban low-rise (Meas.LOS-Lb-btHouse) & & 3.07 & 41.0 \\
\hline & $260-515$ & Urban low-rise (Meas.NLOS-Lb-btHouse) & NLoS & 3.16 & 56.1 \\
\hline
\end{tabular}

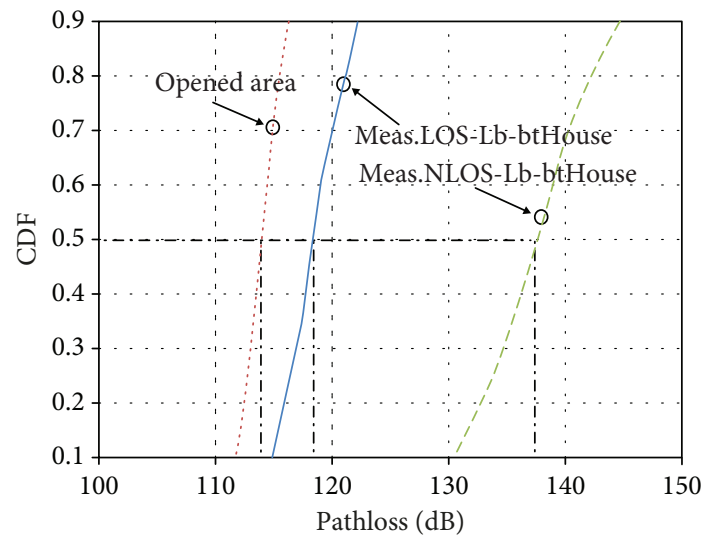

Figure 10: CDF on a path coming from inter-house space (at sitespecific).

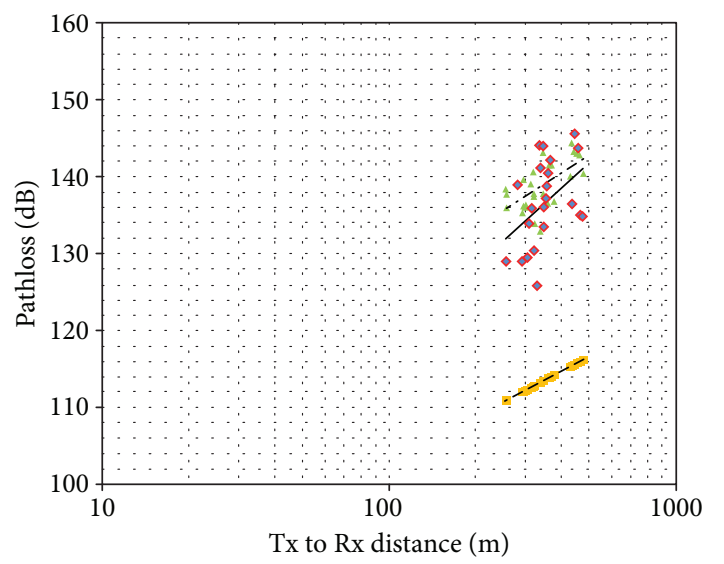

$\Delta$ Meas.NLOS-Lv-over House Roof
$\Delta$ Meas.NLOS-Lb-bt House
Opened area

FIgURE 11: Pathloss characteristics according to the distance on a route that passes over the house roof (at site-specific).

propagation characteristics of a simple and real environment by deriving the propagation characteristic index parameter (attenuation coefficient) based on the measurement. However, since the proposed model is based on actual measurement, it is necessary to derive the propagation loss index parameter based on the optimal measurement according to various environments. Also, it is required to build a measurement system for precise measurement.

4.2. Site-General Characteristics. For the situation where Tx is on the roof and $\mathrm{Rx}$ is below the roof, the proposed sitespecific prediction model is based on the measurement which was performed at multiple $\mathrm{Rx}$ locations with the aim of collecting statistics on the coefficients of propagation. With this prediction model, it is possible to understand the realistic pathloss characteristic in three routes that pass over the house roof in small town. The pathloss is subdivided into individual loss model based on three different realistic paths: the path along a road, the path between houses, and the path above the house roof. On the other hand, the measurementbased site-general pathloss model shows the difference with the environment applied for the site-specific model that is divided by the specific environments based on buildings and the road. The site-general model is divided into LoS and NLoS environments according to visibility and is divided into the opened area, urban, suburban, residential, rural area, etc. The propagation pathloss model for calculating the cell coverage and the interference of a real communication system uses the site-general model. At the mm-Wave frequencies above about $30 \mathrm{GHz}$, the breakpoint distance far exceeds the expected maximum cell radius of 500 meters. The power distance attenuation factor is almost pathloss exponents of about 1.9 to 4.0 . The site-general model was applied to the situation. ITU-R recommends a radio propagation characteristic model (Recommendation ITU-R P. 1411) that can be used from $300 \mathrm{MHz}$ to $100 \mathrm{GHz}$ for the short-range communication within $1 \mathrm{~km}$. The recommendation provides a site-general propagation model for over rooftop propagation in the urban environment. Applied frequency and the application of the distance and environment are different according to visible (LoS) or invisible (NLoS) area. Figure 13 shows pathloss according to the distance from the Tx site to $\mathrm{Rx}$ by the measurement (Meas.LOS-Suburban) or the existing prediction model (P.1411 LOS-Urban) result for over rooftop paths in the LoS environment. Figure 14 shows pathloss according to the distance by the measurement (Meas.NLOS-Suburban) result or the existing prediction model (P.1411 NLOS-Urban) result for the over rooftop paths in the NLoS environment. The regression lines in LoS and NLoS environments were drawn based on the measurement data.

Table 5 shows the coefficients $\left(\alpha_{s g}, \delta_{s g}\right)$ derived from the Figures 13 and 14.

The coefficients associated with pathloss are different depending on the LoS or NLoS environment. That is, in the case of NLoS, the relative pathloss is greater than LoS, and the slope with respect to pathloss also increases sharply. The pathloss factor according to the distance seems larger than the opened area loss in case of LoS, and relative pathloss in NLoS is about $23 \mathrm{~dB}$ larger than that in LoS. Experimental-based pathloss characteristics at $32.4 \mathrm{GHz}$ bands for the over rooftop in a small town (a kind of suburban) were compared with the over rooftop in the urban 
Table 3: Pathloss coefficient on a route that passes over the house roof (at site-specific).

\begin{tabular}{|c|c|c|c|c|c|}
\hline Frequency $(\mathrm{GHz})$ & Distance range $(\mathrm{m})$ & Type of environment & LoS/NLoS & $\alpha_{3}$ & $\delta_{3}$ \\
\hline \multirow{2}{*}{32.4} & \multirow{2}{*}{$260-480$} & Opened area & LoS & 2.0 & 62.7 \\
\hline & & Urban low-rise (Meas.NLOS-Lv-over House Roof) & NLoS & 2.42 & 77.5 \\
\hline
\end{tabular}

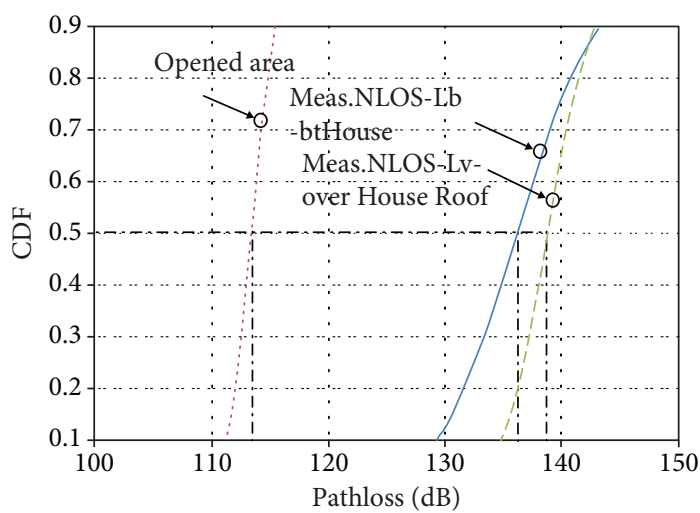

Figure 12: CDF on a route that passes over the house roof (at sitespecific).

TABLE 4: Estimation errors of proposed model in suburban environments.

\begin{tabular}{lccc}
\hline Environment & & RMSE & \\
& $L_{r}(\mathrm{~dB})$ & $L_{b}(\mathrm{~dB})$ & $L_{v}(\mathrm{~dB})$ \\
\hline LoS & 2.7 & 0.7 & - \\
NLoS & 3.9 & 4.7 & 2.4 \\
\hline
\end{tabular}

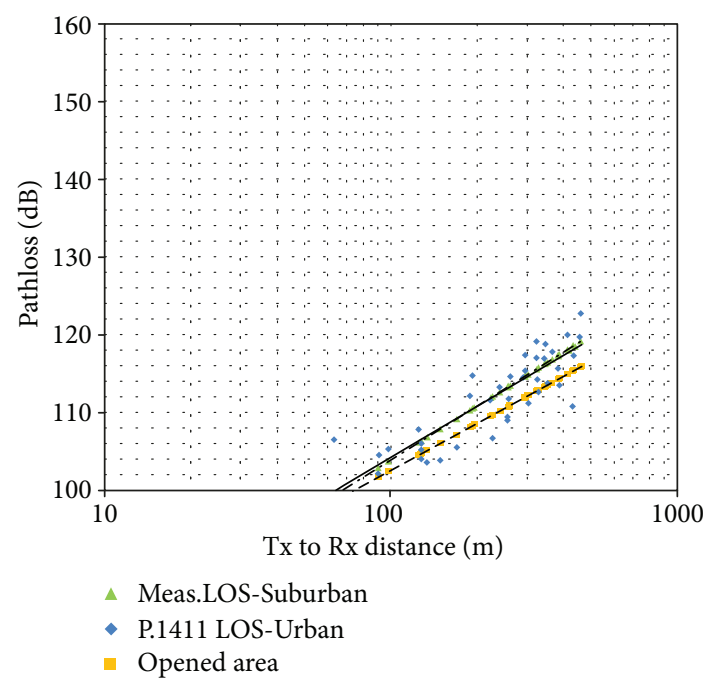

Figure 13: Pathloss characteristics according to the distance in LoS environment (at site-general).

environment of recommendation ITU-R P.1411 and results of the opened area characteristics at the same frequency bands. The experimental analysis is based on the fact that the pathloss model for the over rooftop in an urban lowrise (suburban) environment exists in the visible region and in the invisible region. Therefore, it is possible to identify the similarities with existing models in the visible region

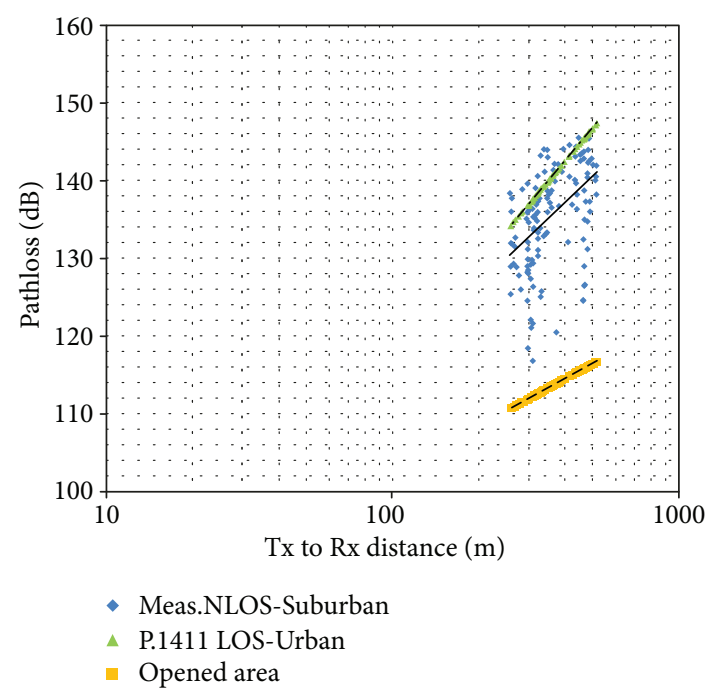

FIgURE 14: Pathloss characteristics according to the distance in NLoS environment (at site-general).

through the analysis of actual measurements and to present pathloss characteristics of the suburban environment in the invisible region as the basis for the measurement. It was judged to be available. As a result in Table 5, it is confirmed that the attenuation index $\left(\alpha_{s g}\right)$ according to the distance in the visible region at $32.4 \mathrm{GHz}$ bands for the over rooftop in a small town environment is 2.2 , which is similar to the existing model with an attenuation coefficient of 2.3.

The attenuation index according to the distance in the invisible region is 3.5 ; it is less than 4.4 of the existing urban model of recommendation ITU-R P.1411. Typical pathloss parameters estimated from all power profiles of the path in the LoS and NLoS environment for the whole measurement area are given in Table 5 . The probability values of $50 \%$ CDF for pathlosses at $32.4 \mathrm{GHz}$ in small town are shown in Figure 15 in LoS and Figure 16 in NLoS, respectively. On the site-general characteristics in a small town of a suburban area, median pathloss values of the opened area, LoS, and NLoS environments are $111 \mathrm{~dB}, 112 \mathrm{~dB}$, and $135 \mathrm{~dB}$, respectively.

\section{Conclusions}

In this paper, we propose the measurement basis of the sitespecific pathloss model for the over rooftop paths in a small town and also provide the site-general characteristics in the entire measurement area of a small town at the mm-Wave frequency bands. In the case of the over rooftop route, the propagation is transmitted from the $\mathrm{Tx}$ site to $\mathrm{Rx}$ in three paths according to the environment of the road and buildings in a small town. One is along the road path, the second 
TABLE 5: Pathloss coefficient in LoS and NLoS environment (at site-general).

\begin{tabular}{|c|c|c|c|c|c|}
\hline Frequency $(\mathrm{GHz})$ & Distance range $(\mathrm{m})$ & Type of environment & LoS/NLoS & $\alpha_{s g}$ & $\delta_{s g}$ \\
\hline \multirow{6}{*}{32.4} & \multirow{3}{*}{$63-461$} & Opened area & \multirow{3}{*}{ LoS } & 2.0 & 46.9 \\
\hline & & Urban high-rise (P. 1411 LOS-Urban) & & 2.3 & 58.2 \\
\hline & & Urban low-rise (Meas.LOS-Suburban) & & 2.2 & 59.9 \\
\hline & \multirow{3}{*}{ 257-514 } & Opened area & \multirow{3}{*}{ NLoS } & 2.0 & 62.6 \\
\hline & & Urban high-rise (ITU-R P. 1411 NLOS-Urban) & & 4.4 & 28.5 \\
\hline & & Urban low-rise (Meas.NLOS-Suburban) & & 3.5 & 46.9 \\
\hline
\end{tabular}

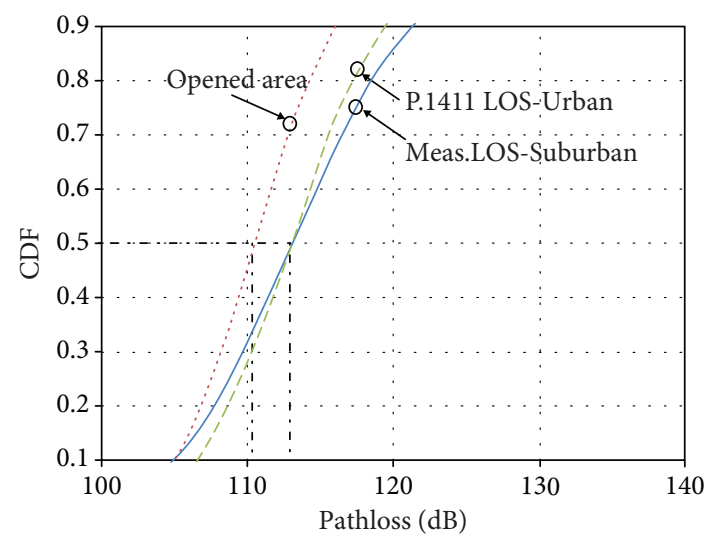

Figure 15: CDF in LoS environment (at site-general).

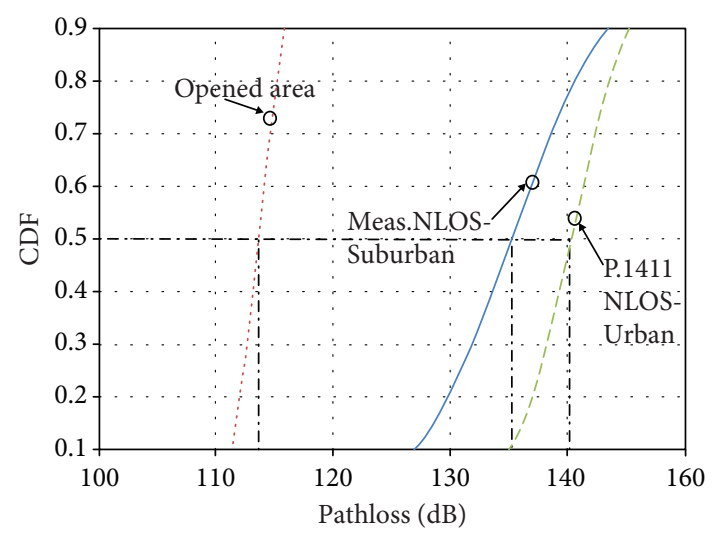

FIGURE 16: CDF in NLoS environment (at site-general).

is between houses, and the third is the roof path. In the case of the existing ITU-R pathloss model, only the site-specific model for the roof path out of various paths was considered, but there was a limit to reflect various small urban environments. In addition, although the site-general model is used as the propagation model for the system cell coverage calculation and frequency sharing between heterogeneous systems; there is no reliable suburban model in the mm-Wave bands. Therefore, the site-specific prediction model based on measurement and geometry that can reflect the characteristics of a small town in a suburban environment is proposed and the pathloss coefficient factor in the suburban environment is presented. These results can be very useful as a deterministic pathloss and clutter loss models in the elevation link for sharing research based on cell planning and frequency shortage according to the deployment of a cellular environment of future $5 \mathrm{G}$ service systems or the earth-space (satellite) link at millimeter wave bands.

\section{Data Availability}

The data used to support the findings of this study are available from the corresponding author upon request.

\section{Conflicts of Interest}

The authors declare that they have no conflicts of interest.

\section{Acknowledgments}

This work was supported by Institute for Information \& Communications Technology Planning \& Evaluation (IITP) grant funded by the Korean government (MSIT) (No. 2017-000066, "Development of time-space based spectrum engineering technology for the preemptive using of frequency").

\section{References}

[1] M. R. Akdeniz, Y. Liu, M. K. Samimi et al., "Millimeter wave channel modeling and cellular capacity evaluation," IEEE Journal on Selected Areas in Communications, vol. 32, no. 6, pp. 1164-1179, 2014.

[2] G. R. MacCartney, J. Zhang, S. Nie, and T. S. Rappaport, "Path loss models for $5 \mathrm{G}$ millimeter wave propagation channels in urban microcells," in 2013 IEEE Global Communications Conference (GLOBECOM), pp. 3948-3953, Atlanta, GA, USA, December 2013.

[3] T. S. Rappaport, Wireless Communications, Principles and Practice, Prentice, Prentice Hall, 1996.

[4] J. Lee, M.-D. Kim, J.-J. Park, and Y. J. Chong, "Field-measurement-based received power analysis for directional beamforming millimeter-wave systems: effects of beamwidth and beam misalignment," ETRI Journal, vol. 40, no. 1, pp. 26-38, 2018.

[5] D. He, B. Ai, K. Guan et al., "Stochastic channel modeling for railway tunnel scenarios at $25 \mathrm{GHz}$," ETRI Journal, vol. 40, no. 1, pp. 39-50, 2018.

[6] ITU, Rec. ITU-R P.1411-9, Propagation data and prediction methods for the planning of short-range outdoor radiocommunication systems and radio local area networks in the frequency range $300 \mathrm{MHz}$ to $100 \mathrm{GHz}$, Vol. 2017 P Series, ITU, Geneva, 2017.

[7] European Cooperation in the Field of Scientific and Technical Research EURO-COST231, Urban Transmission Loss Models Mobile Radio in the 900 and $1800 \mathrm{MHz}$ Bands. Rev. 2, COST 
(European COoperative for Scientific and Technical research committee), The Hague, Netherlands, 1991.

[8] Y. Yoon, J. Kim, K. Kim, M. Kim, and Y. Chong, "Clutter loss characteristic in mm-wave bands for small urban environment," in 2017 International Conference on Information and Communication Technology Convergence (ICTC), pp. 12661268, Jeju, South Korea, October 2017.

[9] N. Kita, W. Yamada, and A. Sato, "Path loss prediction model for the over-rooftop propagation environment of microwave band in suburban areas," Electronics and Communications in Japan (Part I: Communications), vol. 90, no. 1, pp. 13-24, 2007.

[10] L. R. Maciel, H. L. Bertoni, and H. N. Xia, "Unified approach to prediction of propagation over buildings for all ranges of base station antenna height," IEEE Transactions on Vehicular Technology, vol. 42, no. 1, pp. 41-45, 1993.

[11] P. Valtr, J. Zeleny, P. Pechac, and M. Grabner, "Clutter loss modelling for low elevation link scenarios," International Journal of Antennas and Propagation, vol. 2016, Article ID 1478471, 4 pages, 2016.

[12] M. M. Ahamed and S. Faruque, "Propagation factors affecting the performance of $5 \mathrm{G}$ millimeter wave radio channel," in 2016 IEEE International Conference on Electro Information Technology (EIT), pp. 728-733, Grand Forks, ND, USA, May 2016.

[13] K. Amjad, M. Ali, S. Jabbar, M. Hussain, S. Rho, and M. Kim, "Impact of dynamic path loss models in an urban obstacle aware ad hoc network environment," Journal of Sensors, vol. 2015, Article ID 286270, 8 pages, 2015.

[14] Y.-S. Song and H.-K. Choi, "Analysis of V2V broadcast performance limit for WAVE communication systems using two-ray path loss model," ETRI Journal, vol. 39, no. 2, pp. 213-221, 2017.

[15] J. Lee, J. Liang, M.-D. Kim, J.-J. Park, B. Park, and H. K. Chung, "Measurement-based propagation channel characteristics for millimeter-wave 5G Giga communication systems," ETRI Journal, vol. 38, no. 6, pp. 1031-1041, 2016.

[16] A. I. Sulyman, A. T. Nassar, M. K. Samimi, G. R. Maccartney, T. S. Rappaport, and A. Alsanie, "Radio propagation path loss models for $5 \mathrm{G}$ cellular networks in the $28 \mathrm{GHz}$ and $38 \mathrm{GHz}$ millimeter-wave bands," IEEE Communications Magazine, vol. 52, no. 9, pp. 78-86, 2014.

[17] I. Rodriguez, H. C. Nguyen, T. B. Sorensen et al., "Analysis of $38 \mathrm{GHz}$ mmWave propagation characteristics of urban scenario," in Proceedings of European Wireless 2015; 21th European Wireless Conference, pp. 374-381, Budapest, Hungary, May 2015.

[18] H. Xu, T. S. Rappaport, R. J. Boyle, and J. H. Schaffner, "Measurements and models for $38-\mathrm{GHz}$ point-to-multipoint radiowave propagation," IEEE Journal on Selected Areas in Communications, vol. 18, no. 3, pp. 310-321, 2000.

[19] P. kyosti, I. Carton, and A. Karstensen, "Frequency dependency of channel parameters in urban LOS scenario for mmWave communications," in 2016 10th European Conference on Antennas and Propagation (EuCAP), Davos, Switzerland, April 2016.

[20] A. Seville, P. G. V. Charrier, and N. Powell, "Building scatter and vegetation attenuation measurement at $38 \mathrm{GHz}$," in Ninth International Conference on Antennas and Propagation (ICAP), pp. 46-50, Eindhoven, Netherlands, April 1995.

[21] H. Zhao, R. Mayzus, S. Sun et al., " $28 \mathrm{GHz}$ millimeter wave cellular communication measurements for reflection and penetration loss in and around buildings in New York City," in 2013 IEEE International Conference on Communications (ICC), pp. 5163-5167, Budapest, Hungary, June 2013.

[22] P. A. Tenerelli and C. W. Bostian, "Measurements of $28 \mathrm{GHz}$ diffraction loss by building corners," in Ninth IEEE International Symposium on Personal, Indoor and Mobile Radio Communications (Cat. No.98TH8361), pp. 1166-1169, Boston, MA, USA, September 1998.

[23] ITU, Rec. ITU-R P.525-3, Calculation of Free-Space Attenuation, ITU-R Recommendation, Vol. 2016 P Series, ITU, Geneva, 2016. 


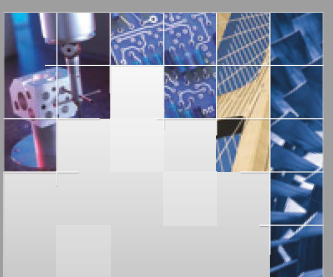

\section{Enfincering}
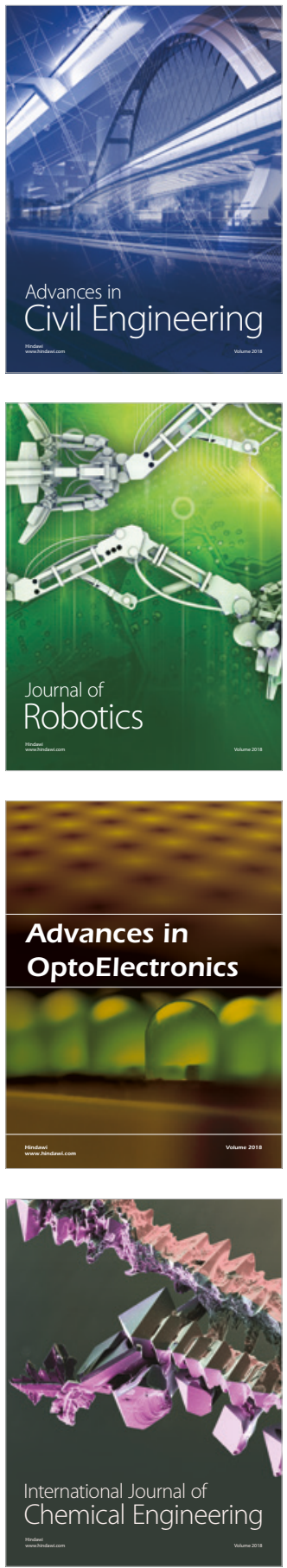

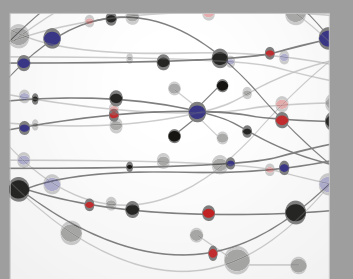

\section{Rotating \\ Machinery}

The Scientific World Journal

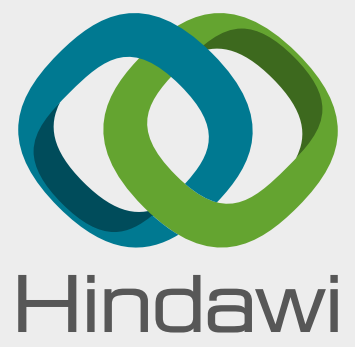

Submit your manuscripts at

www.hindawi.com
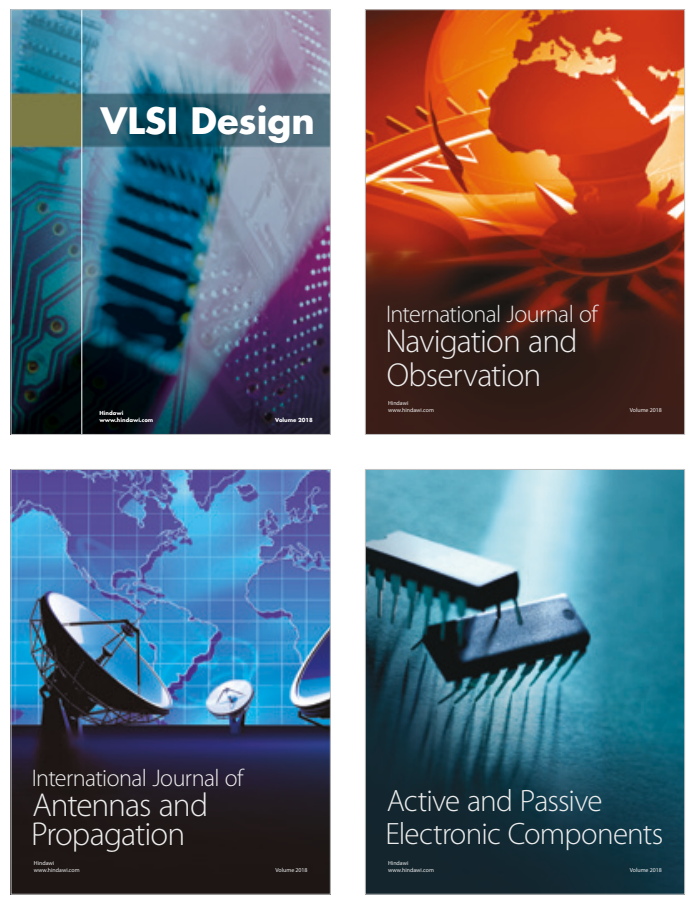
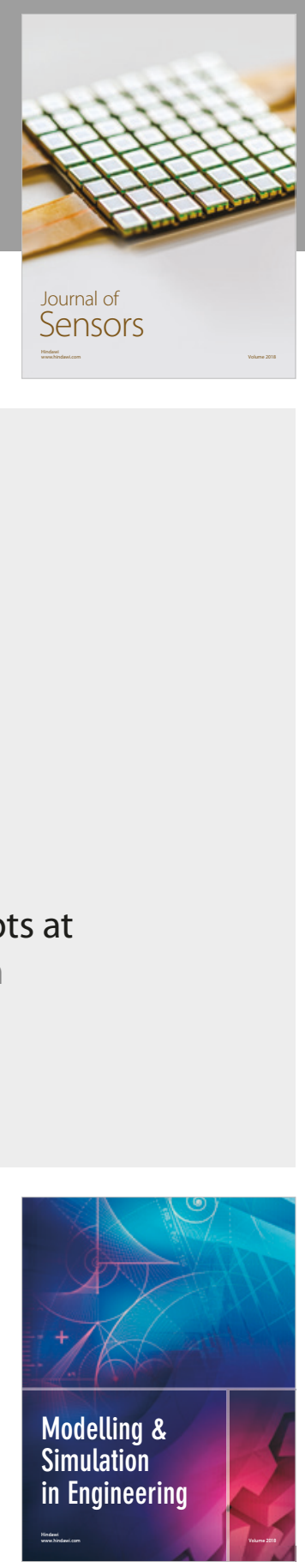

\section{Advances \\ Multimedia}
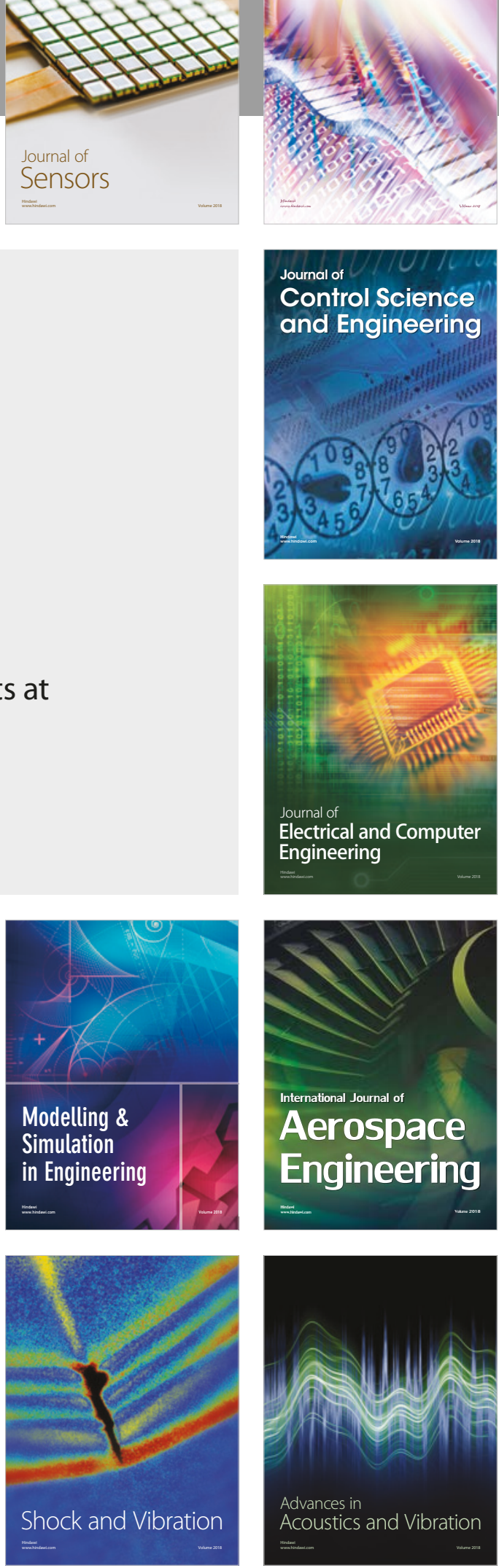